\title{
Mesures par anémométrie laser derrière des corps bi- et tridimensionnels
}

\author{
Laser velocity measurements \\ behind two and three dimensional bodies \\ B.C. Jaeggy \\ C. Berner \\ Institut franco-allemand de recherches de Saint-Louis
}

\begin{abstract}
Le but de cet article est d'illustrer par quelques exemples significatifs l'intérêt de l'anémométrie laser pour l'étude des écoulements décollés et en particulier pour l'analyse des caractéristiques instationnaires et turbulentes de ces écoulements. On ne rappellera donc pas ici les principes de la méthode qui sont maintenant bien connus et on ne décrira pas les montages employés, au sujet desquels on voudra bien se reporter à la bibliographie. Les exemples passés en revue concernent la transition par décollement laminaire et recollement turbulent sur un profil d'aile. la couche limite turbulente proche du décollement sur le même profil, l'allée de tourbillons derrière un profil d'aile muni d'un spoiler et enfin le champ d'écoulement derrière un culot cylindrique droit et un culot avec méplats.
\end{abstract}

The purpose of this paper is to present, by means of a few significant examples, the benefits of laser Doppler velocimetry $(L D V)$ in the study of the unstationnary and turbulent characteristics in separated flows. The principles of the method is now well known and therefore the LDV systems mentionned in the references will not be described in this paper. Experimental investigations concern the study of the laminar-turbulent transition on an airfoil as well as the turbulent boundary layer near the separation, the vortex street behind an airfoil equipped with a spoiler, and finally the flow field behind a cylindrical afterbody and a triangular boattail.

Le département d'aérodynamique de l'Institut francoallemand de recherches de Saint-Louis exploite un certain nombre d'installations d'essais, qui sont en général de dimensions modestes, mais dont le manque de taille est compensé par un équipement en moyens de mesures très supérieur à ce que l'on trouve en général sur des installations analogues. C'est ainsi que deux de ces installations sont munies en permanence de montages d'anémométrie laser bi- et tridimensionnels qui sont utilisés pour l'étude des écoulements décollés et plus particulièrement des caractéristiques instationnaires et turbulentes de ces écoulements.

La première de ces deux installations est une soufflerie subsonique à retour et à veine ouverte de $70 \mathrm{~cm} \times 90 \mathrm{~cm}$, dans laquelle sont essentiellement étudiés des écoulements autour de profils d'aile et donc bidimensionnels.

Le premier exemple d'application qui sera évoqué est celui du profil NACA $65,213 \mathrm{a}=0,5$. C'est un profil ancien, dont l'intérêt pratique actuel est certes limité, mais dont la couche limite présente des caractéristiques telles qu'elle permet de bien mettre en évidence l'intérêt et les possibilités de l'anémométrie laser. En effet, sur ce profil et au nombre de Reynolds considéré, la couche limite reste laminaire jusqu'après le maitre couple puis décolle avant de recoller en turbulent en enfermant une zone de recirculation assez étendue en longueur, mais de faible hauteur.

LA HOUILLE BLANCHE/No 7/8-1987 


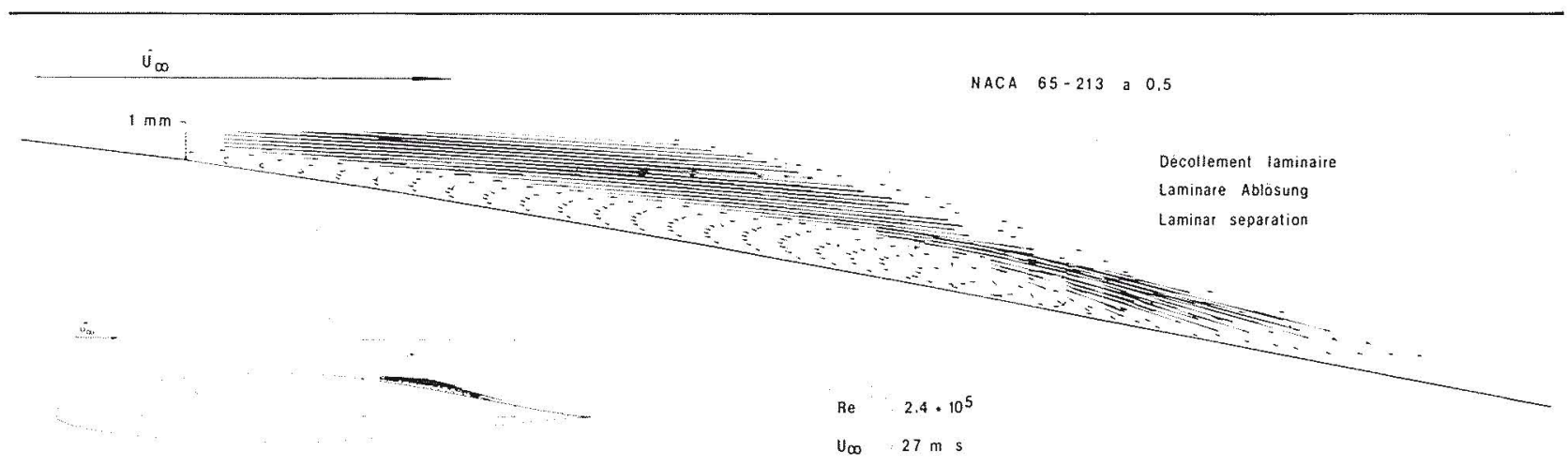

1. Transition par décollement laminaire suivi de recollemem iurbulent $R e=240000, \mathrm{r}_{s \infty}=27 \mathrm{~m} / \mathrm{s}$

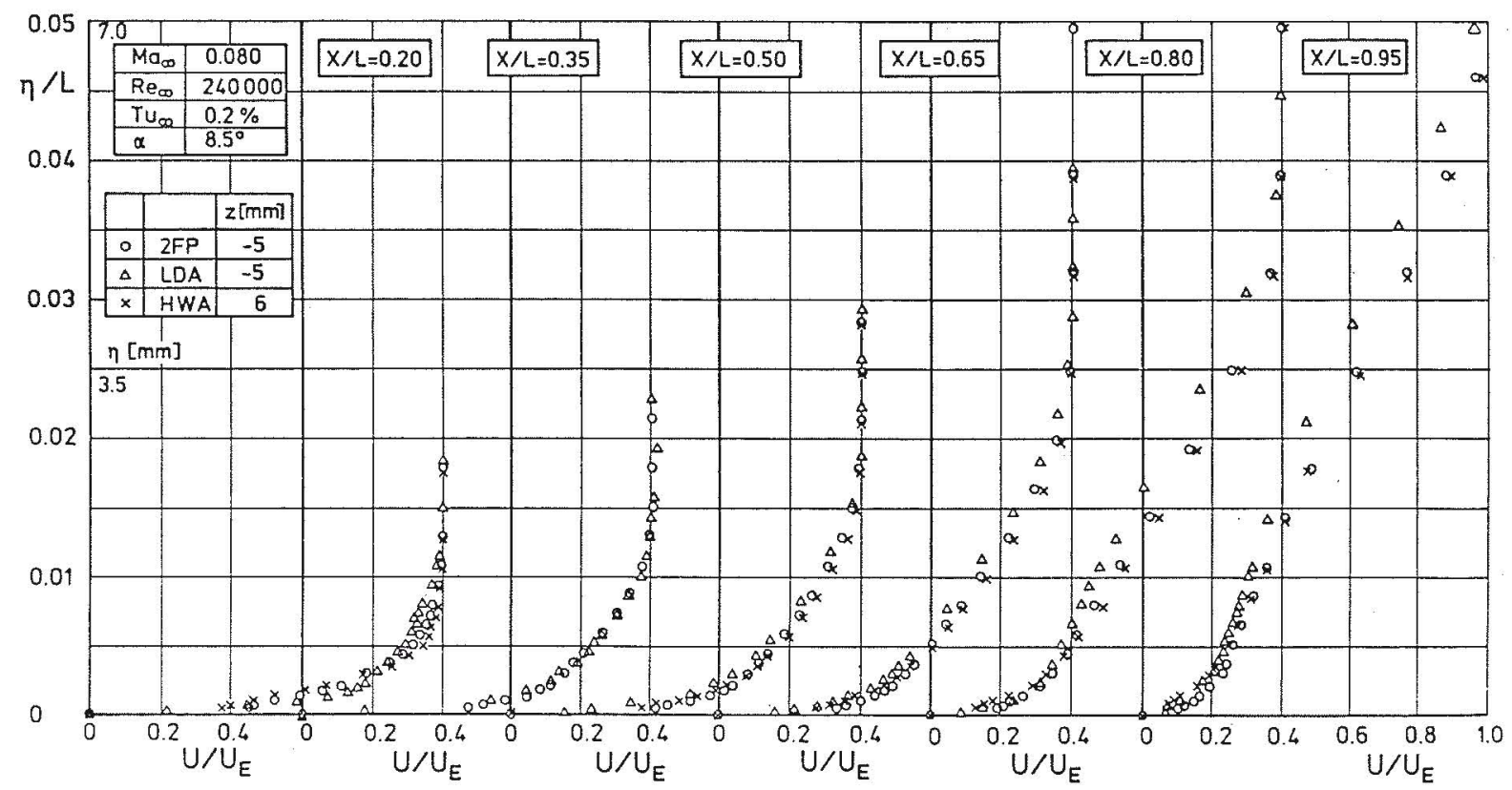

2. Profils de vitesse mesurés sur le profil NACA 65,213a $=0,5$ en incidence. $2 F P=$ sondes de pression; $\angle D A$ : anémométrie laser; HWA : anémométrie à fil chaud.

La figure $I$ donne une représentation des vecteurs vitesses mesurés dans cette zone. L'échelle permet d'apprécier la finesse des mesures qui, dans ce cas, ont pu être poursuivies jusqu'au voisinage immédiat de la paroi.

Sur le même profil, mais placé à une incidence de $8,5^{\circ}$, ia transition se fait toujours par décollement laminaire suivi de recollement turbulent, mais très près du bord d'attaque. La couche limite à l'extrados est donc presque entièrement turbulente, mais le gradient de pression défavorable a tendance à la faire décoller près du bord de fuite. Dans cette configuration, on a procédé [5] à une comparaison des résultats fournis par des sondes de pression, l'anémométrie à fil chaud, l'anémométrie laser et une méthode de calcul de la couche limite turbulente utilisant un modèle de turbulence en $k-\varepsilon$ (modifié pour tenir compte des gradients de pression défavorables)".

La figure 2 donne des exemples de profils de vitesse obtenus à différentes abscisses. Peu après le recollement turbulent $(X / L=0,20)$, on constate des écarts qui disparaissent ensuite, et les résultats expérimentaux fournis par les différentes méthodes sont en bon accord à $X / L$ $=0,35$ et $X / L=0,50$. Plus en aval, les résultats fournis par l'anémométrie laser diffèrent à nouveau de ceux fournis par les deux autres méthodes, ce qui témoigne des perturbations apportées à l'écoulement par les sondes et leur support. Les profils de vitesse calculés n'ont pas été représentés pour ne pas diminuer la lisibilité de la figure.

Ils coïncident sensiblement avec les profils mesurés jusqu'à $X / L=0,80$. Au-delà le calcul n'a pu être poursuivi car le modèle utilisé ne prévoit pas le décolle- 

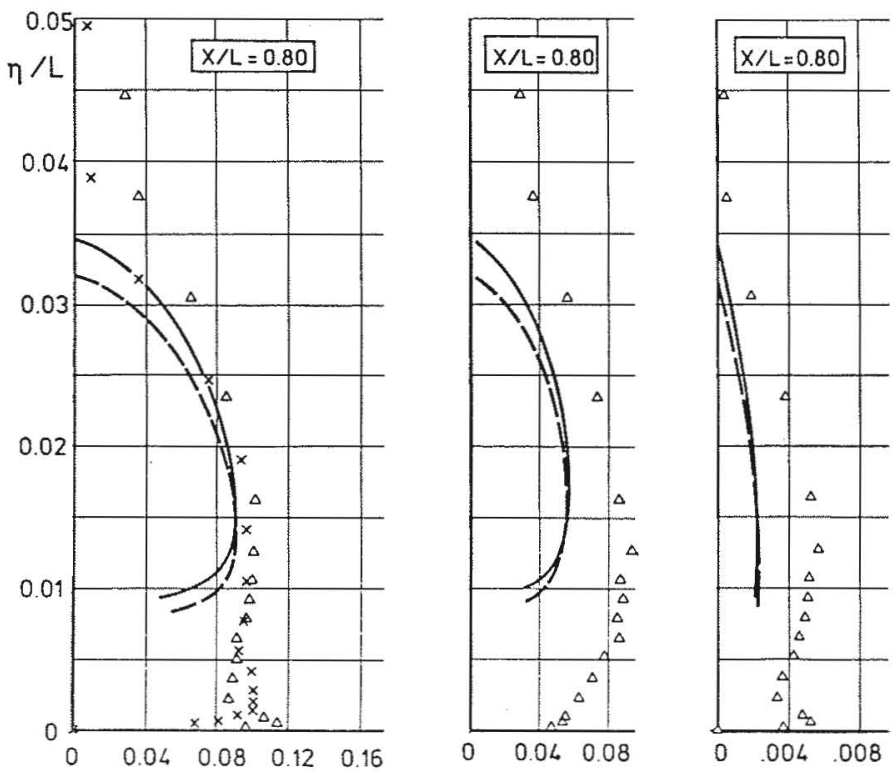

3. Grandeurs turbulentes à $X / L=0,80 \cdot \sqrt{\overline{u^{\prime 2}}} / v_{r} ; \cdot \sqrt{\sqrt{v^{\prime 2}}} / v_{p}$; $-\overline{u^{\prime} v^{\prime}} / v_{e}^{2}$.

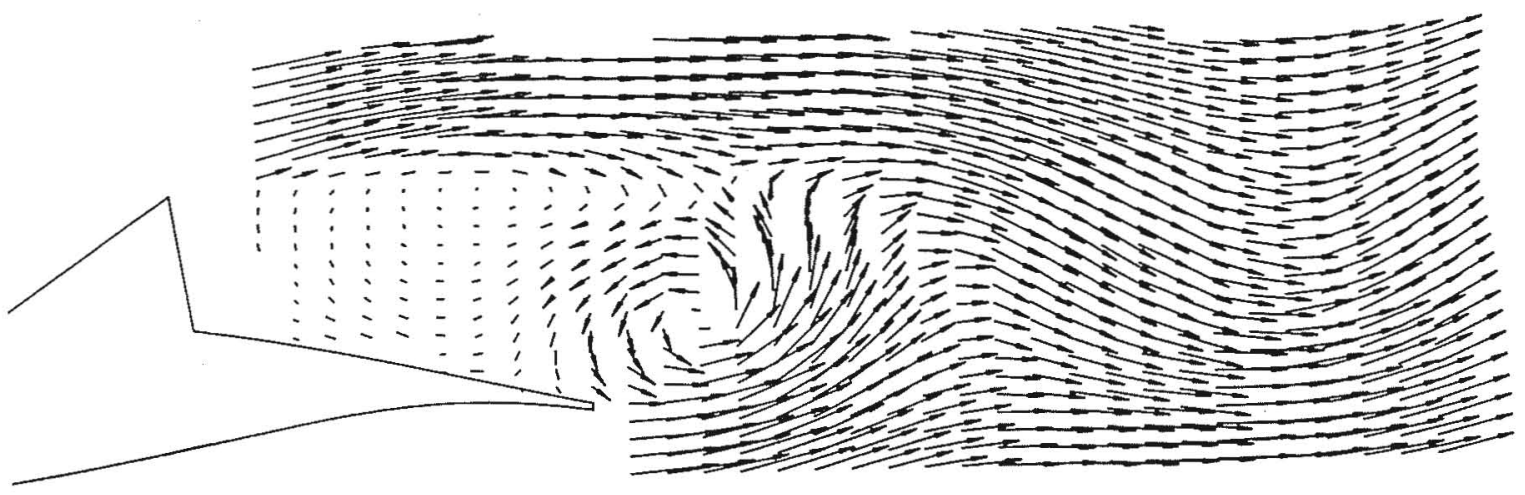

4. Exemple de champ de vitesses instantanées. Profil RA 16 SC 1 muni d'un spoiler à $40^{\circ}$. $v_{\infty}=35 \mathrm{~m} / \mathrm{s}, R e=400000$. Axes liés au profil.

ment turbulent.

La figure 3 donne le taux de turbulence longitudinal, le taux de turbulence transversal et la tension de Reynolds à $X / L=0,80$. L'accord entre l'expérience et le calcul est dans l'ensemble acceptable (deux calculs ont été effectués en prenant comme données initiales soit les résultats de mesures par anémométrie laser, soit les résultats des mesures au fil chaud à $X / L=0,20$ ).

Le deuxième exemple est celui de l'étude de l'allée de tourbillons qui se développe derrière le profil supercritique Aérospatiale RA 16 SC 1 muni d'un spoiler d'extrados. Comme il s'agit d'un écoulement instationnaire, il faut, dans ce cas, mesurer non seulement les composantes de la vitesse, mais aussi la phase de chaque mesure individuelle (la mesure est déterminée aléatoirement par le passage d'une particule dans le volume de mesure) par rapport à la période de l'allée de tourbillons. Le traitement des données brutes a été fait en divisant la période de l'allée de tourbillons en 20 intervalles. On en trouvera la description en [4] ou en [6]. La figure 4 donne un exemple de champ de vitesses instantanées ainsi obtenu. On y distingue nettement la formation du tourbillon émis au bord de fuite du profil. Les lignes d'émission obtenues par le calcul à partir des champs de vitesses mesurées sont plus parlantes.

* Ce travail a été effectué en coopération avec l'Institut für Entwurfsaerodynamik de la Deutsche Forschungs- und Versuchsanstalt für Luft- und Raumfahrt à Braunschweig. 

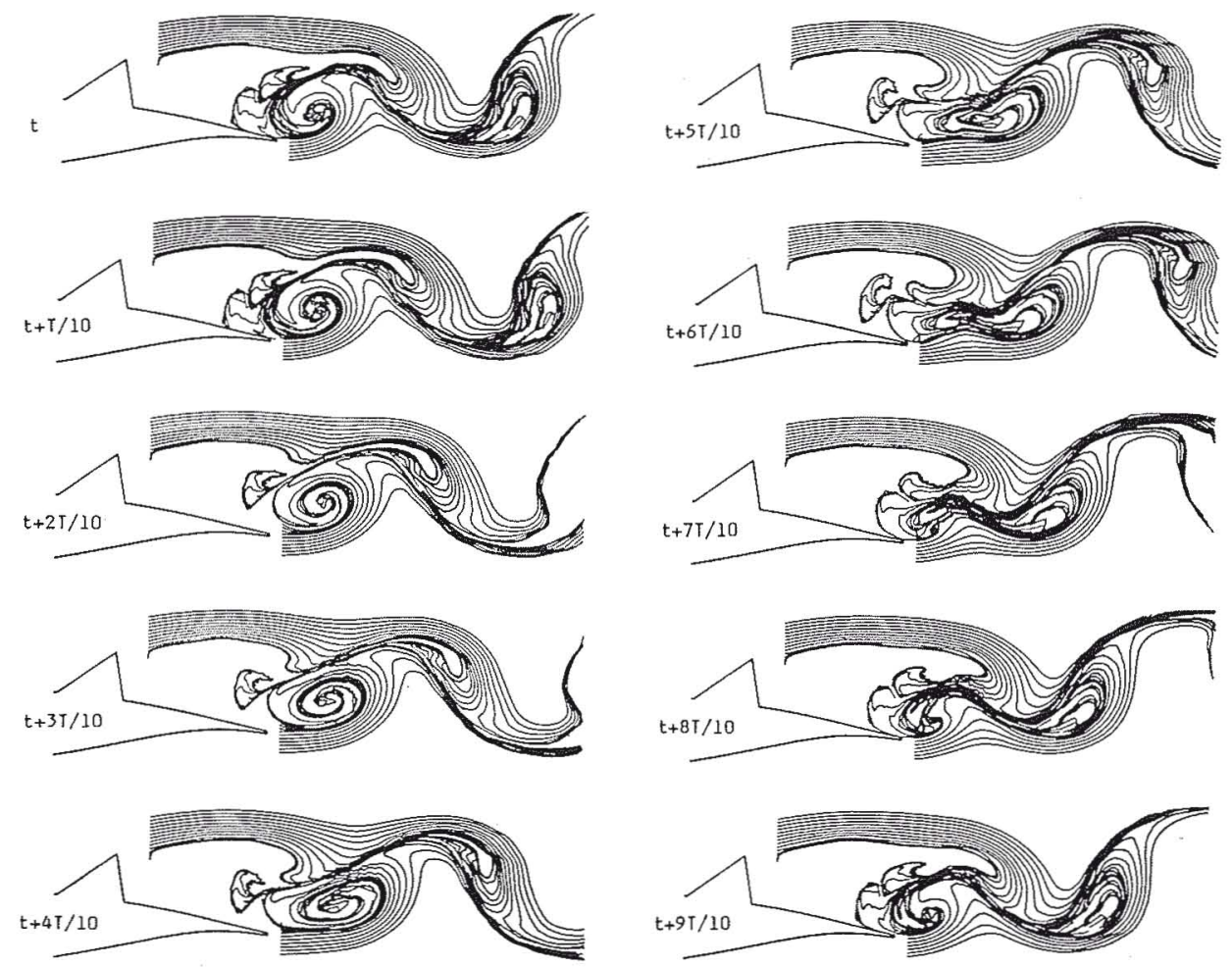

5. Evolution du champ des lignes d'émission pendant une période de l'allée de tourbillons. Profil RA I6 SC 1 avec spoiler d'extrados braqué à $40^{\circ} . v_{\infty}=35 \mathrm{~m} / \mathrm{s}, R e=400000$.

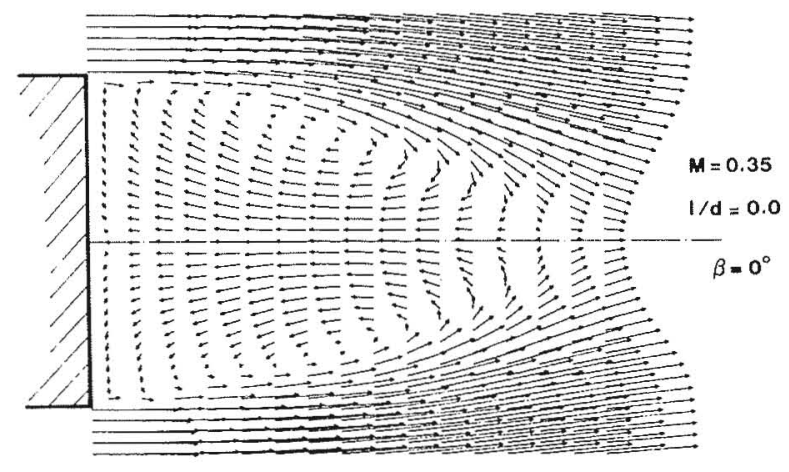

6. Champ de vitesses.

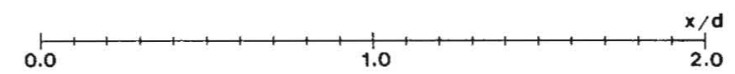

La figure 5 donne l'évolution de ces lignes pour une période. Là, on voit très nettement la formation et le développement du tourbillon de bord de fuite, sa déformation progressive sous l'action du tourbillon issu de l'arête du spoiler puis son détachement et sa convection vers l'aval alors que se développe un nouveau tourbillon de bord de fuite. La nappe tourbillonnaire issue de l'arête du spoiler suit une évolution analogue, à ceci près que son enroulement ne se fait qu'à une certaine distance en aval.

Les lignes d'émission d'un écoulement sont ce que montrent des visualisations par plan laser. Les images qui précèdent sont donc directement comparables à de telles visualisations [7], mais à la différence de ces dernières, elles ont une valeur quantitative.

La deuxième installation de l'ISL munie en permanence d'un montage d'anémométrie laser est un jet libre permettant d'obtenir des écoulements dont le nombre de Mach est compris entre 0,22 et 0,93 , dans des tuyères de 80 à $120 \mathrm{~mm}$ de diamètre. Cette installation a été utilisée pour l'étude d'écoulements de culot d'arrière-corps de formes variées [1-3].

La figure 6 présente un exemple de champ de vitesses obtenu par anémométrie laser pour un culot cylindrique 


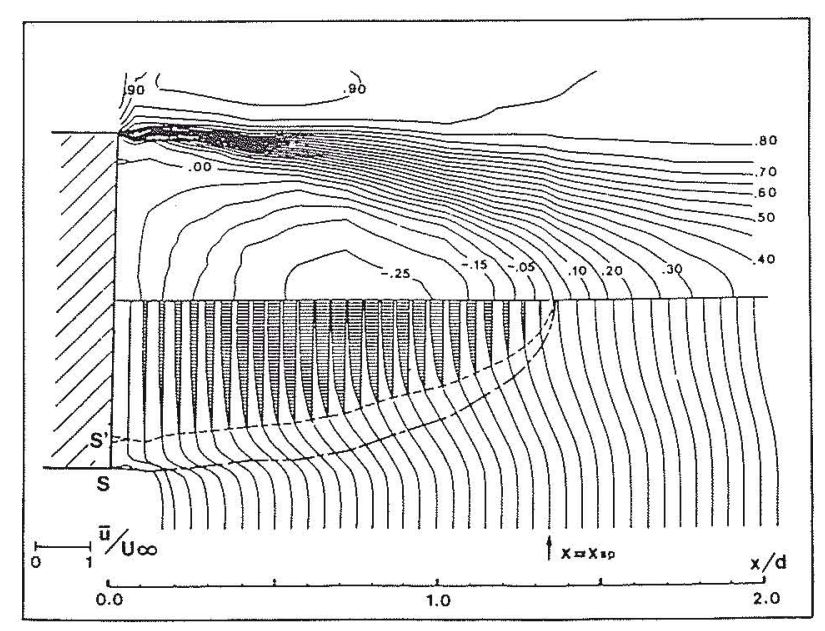

STREAHYISE VELOCITY $\vec{u}(x, y)$

$\left(\beta=0^{\circ}, M=0.35\right)$

7. Lignes iso-vitesse et profils de vitesse longitudinale. Culot droit. $d=20 \mathrm{~mm}, M=0.35, R^{\prime}=150000$.

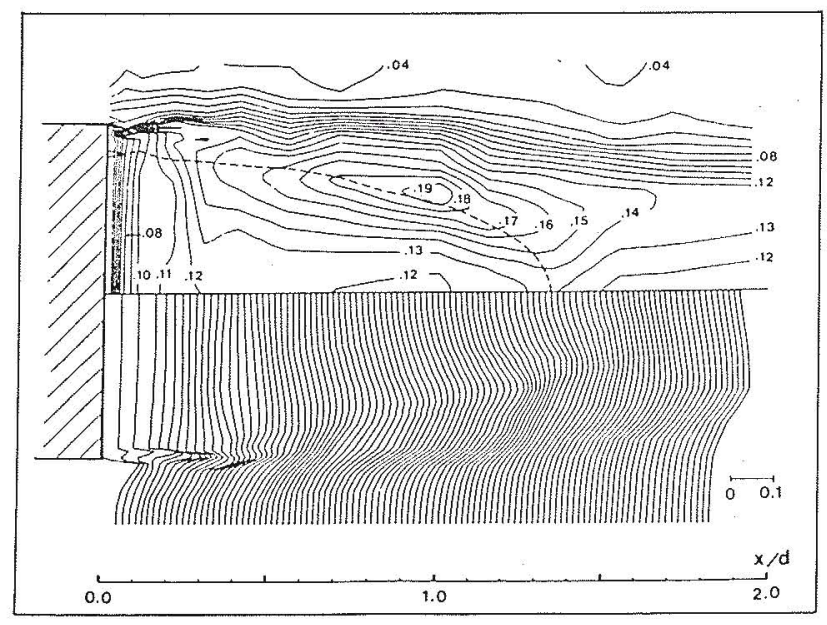

AXIAL TURBULENCE INTENSITY

$\left(\beta=0^{\circ}, M=0.35\right)$

8. Imensités de nurbulence axiale $\cdot \sqrt{\overline{u^{2}}} / \gamma_{\mathrm{cos}}$.

droit [1] et la figure 7 présente pour le même cas les courbes isovitesses (en haut) et les profils de vitesse mesurés (en bas), la partie hachurée correspondant aux zones où la vitesse est négative.

On a également représenté sur cette figure la ligne le long de laquelle la composante $\bar{u}$ de la vitesse est nulle et la ligne de courant issue du point de séparation. On remarquera que la ligne de vitesse nulle arrive sur le culot en un point $S^{\prime}$ qui ne coïncide pas avec l'arête $S$. On a vérifié qu'il ne s'agissait pas là d'un résultat erroné dû soit à l'influence des erreurs de mesure, soit à celle de la

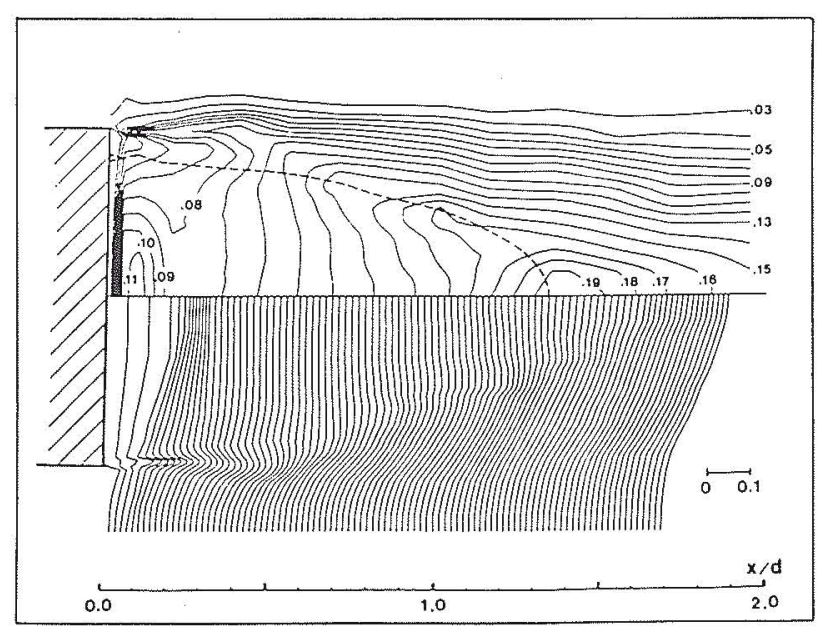

RADIAL TURBULENCE INTENSITY $\left(\beta=0^{\circ}, H=0.35\right)$

9. Intensités de turbulence radiale $\cdot \sqrt{\sqrt{12}} / r_{\infty}$

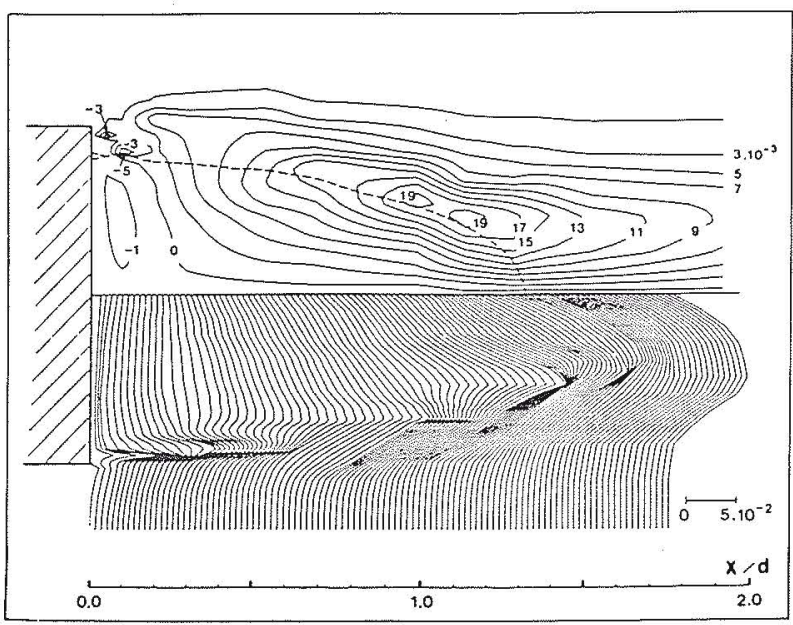

SHEAR STRESS OISTRIBUTIONS

10. Tension de Reynolds $\overline{u^{\prime} w^{\prime \prime} / t_{\infty}^{2}}$ méthode utilisée pour le traitement et l'exploitation des résultats. Pour ce faire, on a effectué des visualisations pariétales en recouvrant le culot d'un enduit coloré. Cet enduit initialement également réparti se rassemble très vite en un anneau de faible épaisseur dont la position coïncide avec celle du point $S^{\prime}$.

Les figures 8 à 10 présentent, toujours pour le même culot droit, les profils (en bas) et les courbes d'égale intensité (en haut) du taux de turbulence longitudinal (fig. 8), du taux de turbulence transversal (fig. 9) et de la tension de Reynolds (fig. 10). 


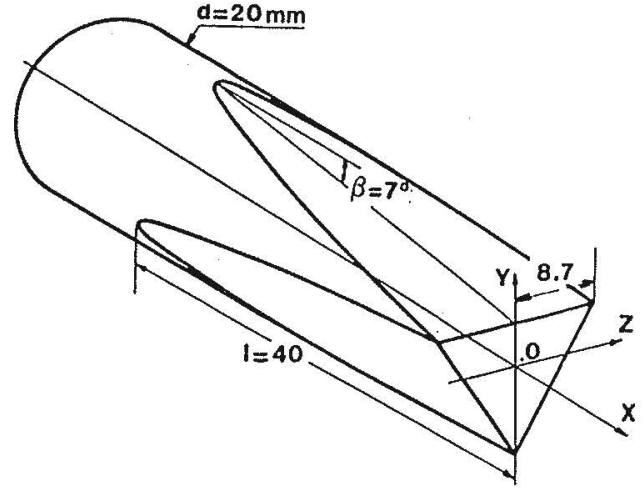

11. Culor avec méplats.

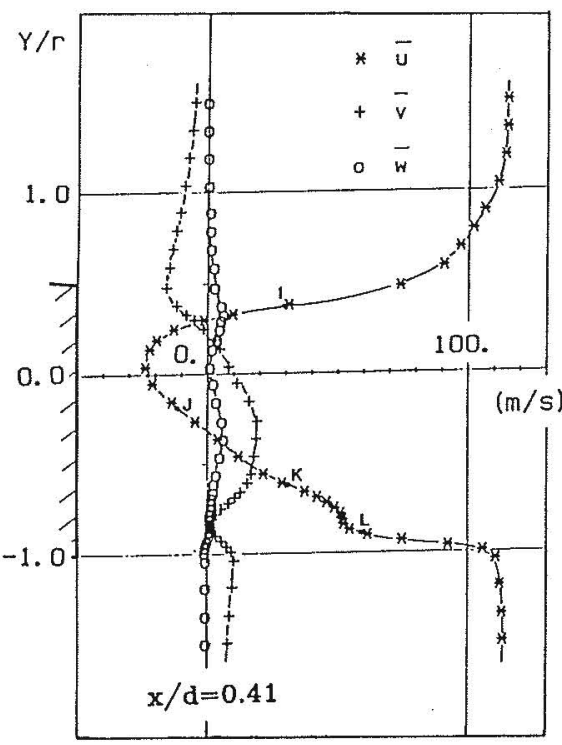

12. Culot avec méplat. Vitesses moyennes à $z=0$.
L'étude du sillage proche d'un culot avec méplats (fig. 11), faite également pour un nombre de Mach de 0,35 et un nombre de Reynolds de 150000 fournit un exemple de résultats concernant un écoulement tridimensionnel [3]. La figure 12 montre l'évolution des composantes de la vitesse moyenne dans le plan de symétrie à 0,41 calibre derrière le culot. On voit la nette dissymétrie entre le haut des courbes, où on se trouve dans le sillage du milieu d'un méplat, et le bas des mêmes courbes, où on se trouve dans le sillage du sommet où se rejoignent deux méplats. Les mesures étant faites dans le plan de symétrie, la composante $\bar{w}$ devrait être rigoureusement nulle, ce qui n'est pas tout à fait le cas. Les valeurs de $\bar{w}$ mesurées sont cependant faibles, et il est bien difficile de dire si elles ont leur origine dans un léger défaut de symétrie du montage, dans le fait que la mesure n'a pas été faite rigoureusement dans le plan de symétrie ou dans un effet tridimensionnel réel. Les figures 13 et 14 donnent, pour la même position, les intensités de turbulence et les valeurs des termes non diagonaux du tenseur de Reynolds. En ce qui concerne les intensités de turbulence, on est frappé par une certaine analogie de la forme des courbes dans leurs parties hautes et dans leurs parties basses malgré une grande différence de niveau.

L'anémométrie laser est une méthode performante et qui est maintenant d'un usage régulier sur nombre de souffleries subsoniques au point de figurer dans la panoplie des méthodes de mesures disponibles sur certaines grandes souffleries (à l'ONERA par exemple). Cependant, elle reste d'une utilisation délicate et sa mise en œuvre ne peut être faite efficacement que par du personnel spécialisé et nécessite une certaine habitude. De plus, elle reste une méthode onéreuse et il n'est pas rare que la valeur d'un montage d'anémométrie laser soit bien plus élevée que celle de la soufflerie sur laquelle il est utilisé. Ceci explique que l'usage de l'anémométrie laser reste limité aux cas tels que les exemples ci-dessus où son emploi est indispensable (écoulements décollés, mesure des éléments du tenseur de Reynolds par exemple). Il serait illusoire de penser que la méthode sera un jour d'un usage aussi courant que le sont par exemple les sondes de pression et qu'elle supplantera totalement ces dernières.

\section{Remerciements}

Les auteurs remercient tous les membres de l'ISL et de la DFVLR, trop nombreux pour être cités en détail ici, qui ont participé aux études dont il a été question dans ce qui précède. 


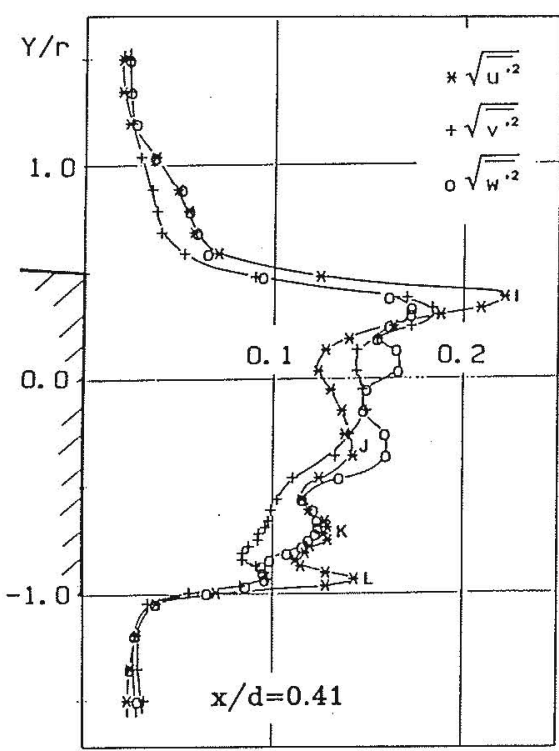

13. Intensités de turbulence à $z=0$.

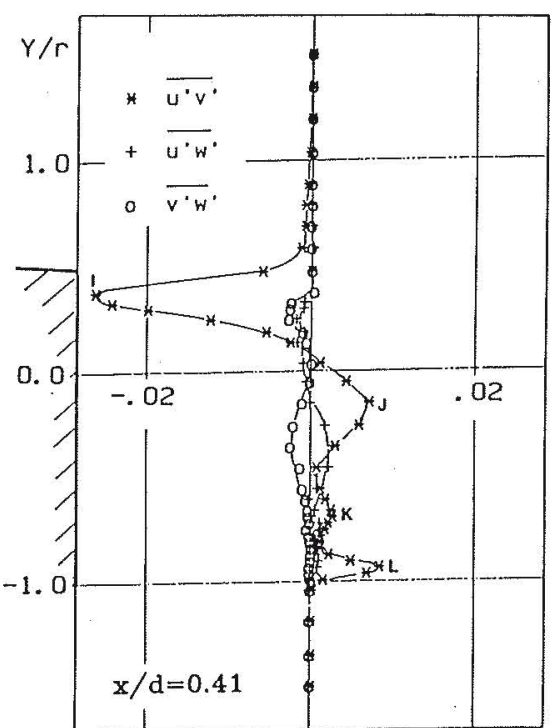

14. Termes non diagonaux du tenseur de Reynolds.

\section{Bibliographie}

[1] C. BERNER, A. KONEKE. - LDA measurements in the turbulent near wake of blunt bodies. 2nd International Symposium on Applications of laser anemometry to fluid mechanics. Lisboa, 2-4 July 1984. ISL - CO 212/84, PU 309/86.

[2] C. BERnER. - Afterbody shape effects on the near-wake velocity characteristics at Mach numbers between 0.35 and 0.92. 38th Meeting of the American Phrsical Society, Division of fluid mechanics, Tucson, Arizona, November 24-29, 1985. ISL - CO 205/86.

[3] C. BERNER. - Simultaneous 3D LDV measurements in the wake of blunt bodies. 3rd International Symposium on Applications of Laser Anemometry to Fluid Mechanics, Lisboa, 7-9 July 1986. ISL - CO 224/86.

[4] B.C. JaegGy, W. Czichowsky, P. Meyer, G. Koerber, R. KauffMAnN. - Contribution à l'étude expérimentale et théorique de l'écoulement autour d'un profil d'aile muni d'un spoiler. L'Aéronautique el l'Astronautique, n 112 (1985), pp. 2-20. ISL - PU 305/86.
[5] B.C. Jaeggy, R. Kauffmann, G. Koerber, R. Kiock, A. Radespiel, H. Hoheisel. - Etude expérimentale de la couche limite sur un profil d'aile par sondes de pression, anémométrie à fil chaud et anémométrie laser et comparaison avec des résultats de calcul. 23' Colloque d'Aérodynamique Appliquée de l'Association Aéronautique et Astronautique de France, Aussois, 12-14 novembre 1986, ISL - CO 241/86 et DFVLR-1 8 129-87/28 (en allemand).

[6] P. Meyer, R. Kauffmann, G. Koerber. - Etude théorique de l'allée altemée de Bénard-Karman. Application à l'étude expérimentale de l'écoulement autour d'un obstacle épais. ISL $-\mathrm{R} 105 / 85$

[7] P. Meyer, L. Madignier, R. KauffmanN. - Visualisation par plan laser de l'allée de tourbillons derrière un profil d'aile muni d'un spoiler: ISL - N 609/85.

\section{Adresse des auteurs}

Messieurs B.C. Jaeggy et C. Berner, Département d'Aérodynamique Institut franco-allemand de recherches de Saint-Louis, 12, rue de l'Industrie, 68301 Saint-Louis 


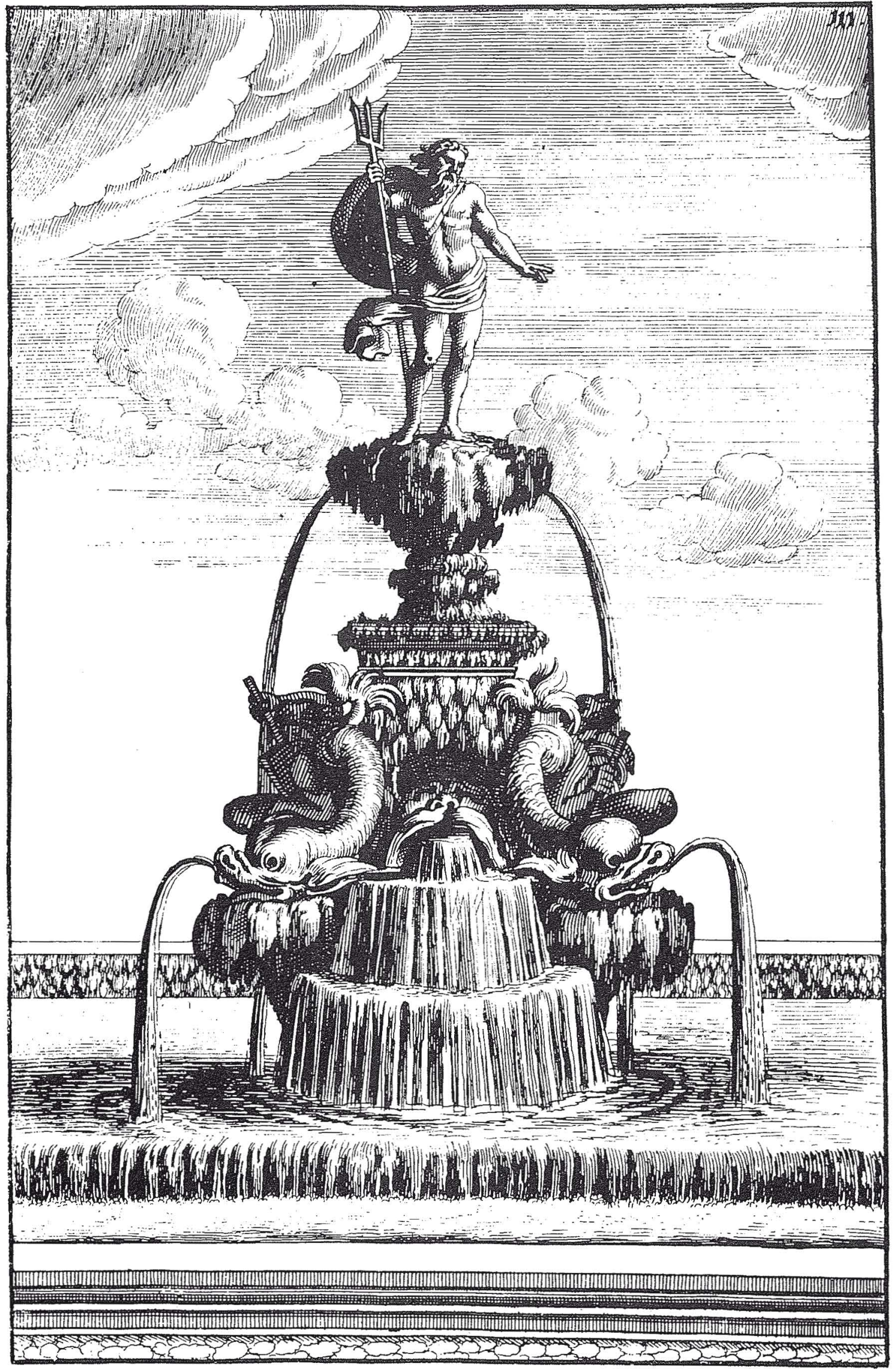

